\title{
Mobile Smart Screening and Remote Monitoring for Vision Loss Diseases
}

\author{
Hussein Khairallah, Lubna Alazzawi, Nabil Sarhan \\ Department of Electrical \& Computer Engineering, \\ Wayne State University, Detroit, Michigan, USA
}

\begin{abstract}
In modern information and communication era, the technology of the smartphone application is one of the most advanced and rapidly developing areas. These applications and its technology in the field of medicine are advancing every day. This demonstrates how it can be used for medical treatment in the field of Ophthalmology. The first aim of this paper is to discuss how a smartphone and its technology can be used to diagnose loss of eye vision. Most recent smartphones have been equipped with a featured camera with high megapixels and advanced sensors which can be used to record fundus photographs through a slit lamp or record videos from an operating microscope and display images from optical coherence tomography systems and other high-tech devices. The ophthalmologists can share these images and analyze with their colleagues by the means of media sharing applications and make the optimal diagnostic and therapeutic results to diagnose the low vision of patients. At present, there are three widely used pocket-sized adapters that can improve the magnification and lighting of the camera, which enables the smartphones to capture high-quality images of the eye. These are the Portable Eye Examination Kit (PEEK), EyeGo, and D-Eye. These adaptors with smartphones have been widely accepted by the medical field for diagnosing low vision and eye-related infections. The second aim of this paper is to provide a meaningful utilization comparison between the smartphone adapters: D-Eye, EyeGo and Portable Eye Examination Kit (PEEK) to distinguish the most suited adapter for vision self-monitoring and screening.
\end{abstract}

Keywords: Diabetic Retinopathy, Cataracts, Glaucoma, Macular Degeneration, Smartphone, Adapter Devices, Portable Eye Examination Kit (PEEK), EyeGo, D-Eye.

\section{INTRODUCTION}

The presence of Smartphones and other portable devices have allowed us to utilize remote healthcare monitoring system for an assortment of structures. Smartphones are quickly turning into the main personal computer and specialized gadget in individuals' lives; they may establish their own network and become the Personal Area Network Coordinator (PANC). Smartphone cameras and applications can alter healthcare by providing doctors with the option to diagnose a patient beyond traditional treatment equipment, as well as being able to assist patients in receiving beneficial information and management of health. In Ophthalmology, images captured with the camera sensor on the smartphone can provide as much useful information as a doctor consultation. We can take advantage of this technology to treat vision loss, a common disease that can occur suddenly or gradually. Vision loss differs from blindness, as blindness occurs at birth whereas vision loss occurs over time. The causes of vision loss could range naturally from conditions affecting the eye to conditions affecting the part of the brain that works the eye. This problem is most familiar with age; in the case of the elderly, glaucoma, cataracts, and other eye diseases are the leading factors of vision loss [1]. This paper discusses how a smartphone and its technology can be used to diagnose loss of vision due to Diabetic Retinopathy, Glaucoma and other eye diseases. Most recent smartphones have been equipped with a featured camera with high megapixels and advanced sensors which can be used to record fundus photographs through a slit lamp or record videos from an operating microscope and display images from optical coherence tomography systems and other high-tech devices. The ophthalmologists can share these images and analyze them with colleagues utilizing media sharing applications, thus ensuring the optimal diagnostic and therapeutic results [2-3]. At present, three widely used adapters can improve the magnification and lighting of the camera, which enables smartphones to capture high-quality images of the eye. These are the Portable Eye Examination Kit (PEEK), EyeGo, and DEye. Peek Adapter consists of a smartphone application and retina adapter which can be clipped onto the device and synchronized with the peek application for sharing and analyzing the images. EyeGo is an adapter intended to allow ophthalmologists to capture high-quality images of the eye using the ophthalmic lens.

D-Eye Adapter is one of the extensively used adapters which yield excellent results. It consists of a portable eye and retinal system that fits onto a smartphone, creating a retinal camera for evaluation and screening of the eye. The medical field has widely accepted these smartphone adapters for diagnosing low vision and eye-related infections. This paper is to provide a meaningful utilization comparison between these three smartphone adapters, Examination Kit (PEEK), EyeGo, and DEye.This paper primarily focuses on the causes and symptoms of vision loss. It is organized into the following sections: after the introduction, section 2 discusses the related work. Section 3 presents the vision loss diseases includes diabetic retinopathy, cataracts and glaucoma. Section 4 and 5 provide information about the three camera adapters, portable eye examination kit (PEEK), EyeGo, and D-Eye. Also, these sections provide a meaningful utilization comparison among these three smartphone adapters. Section 6 will wrap up the work of the previous sections with a conclusion.

\section{RELATED WORK}

For over a decade, there have been advances in the field of smartphone technology. Smartphones can run multiple medical applications. This justifies a significant attraction for physicians, due to the simplicity of the natural and valuable communication and the patient made possible by the simple use of an application, R. Lord et al. [4] Julie Brucker, et al. [5]. 
Eye caregivers have a great many applications at their disposal. Some applications are used for ocular surgery, with the goal of enhancing surgical skills or better patient education. Smartphones have reduced the tools required for examination to a small portable adapter. Although they cannot replace office-based testing equipment, they prove helpful in providing fast and accurate data. Also, they can also provide videos, diagrams, and photographs that are convenient for showing treatment methods, Zvornicanin et al. [6] and Bastawrous et al. [7]. Smartphones have improved the cooperation and examination of patients diagnosed with diabetic retinopathy disease by $50 \%$ to $70 \%$ just due to how much time and money was saved in the process. This is causing regular follow up and examination between the patient and the physician, ultimately leading to enhanced, faster treatment, Silva et al. [8]. A small optical device called D-Eye can be attached magnetically to a smartphone, and, once attached, is used to examine and record photos and videos of the retina. The device uses a wireless connection or other possible means of internet connectivity to make it possible for users to obtain retinal photos in even the most remote locations, Russo et al. [9]. A multi-purpose ophthalmic imaging device for Eye scan proves to be easy to use, and its methods of screening for diabetic retinopathy are accurate, Ting et al. [10]. The use of smartphones instead of a beam-splitter for recordings of various surgical procedures smartphones proved useful in recording ophthalmic surgery videos because they are very convenient, easy to use, and costefficient. All those traits would make smartphones the preferred tool of health professionals for surgical recordings, Vyas et al. [11], Cassie Ludwig, et al. [12].

\section{VISION LOSS DISEASES}

\subsection{Diabetic Retinopathy}

Diabetic Retinopathy represents the most common diabetic eye disease. It occurs when the blood vessels change in the retina, swell and leak fluid. Also, new blood vessels appear on the surface of the retina, a sensitive tissue at the back of the eye that transfers images to the brain through the optic nerve. There are two types of retinas: the peripheral retina, which is responsible for vision through the corners of the eyes; and the macula retina, which is responsible for vision through the center of the eye such as reading a book or dialing a phone. Diabetic retinopathy often results in complete vision loss, and victims do not often notice its effect in its early stages, but only as it progresses. Once it has taken effect, there is no reversing the disease [13]. Figure 1 shows a comparison of healthy vision and abnormal retina of diabetic retinopathy.

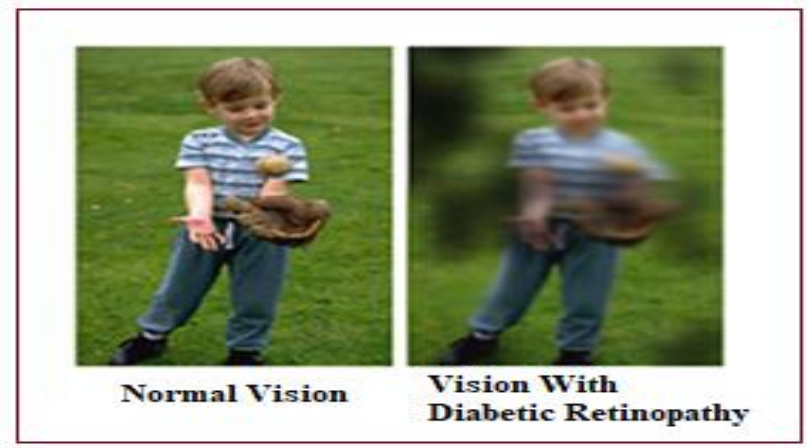

Figure 1: A normal eye and eye with diabetic retinopathy.

\subsubsection{Diabetic Eye Problems}

Diabetic patients could experience two types of Diabetic Retinopathy: Non-proliferative Diabetic Retinopathy (NPDR) and Proliferative Diabetic Retinopathy (PDR). NPDR can cause the following changes in the eye: Microaneurysms, Retinal Hemorrhages, hard Exudates, Macular Edema, and Macular Ischemia. The Microaneurysms are small parts in the blood vessels of the retina that leak fluid. The Retinal Hemorrhage consists of drops of blood that leak into the retina. The Hard Exudates are cholesterol or fats that have leaked into the retina. The Macular edema is the swelling of the Macula due to fluids leaking from the blood vessels. Once it has reached this stage, the retina no longer functions appropriately. Macular edema causes vision loss in people with diabetes. The macular Ischemia occurs when the small blood vessels completely close off, causing blurry vision because the macula no longer receives blood to function correctly. PDR can cause the following changes in the eye: the Vitreous hemorrhage is when blood leaks into the vitreous, which is between the lens and the retina. The Traction Retinal Detachment occurs when the original position of the retina is pulled due to the shrinking of a scarred tissue [14-15]. The two types of diabetic retinopathy are shown in Figure 2.

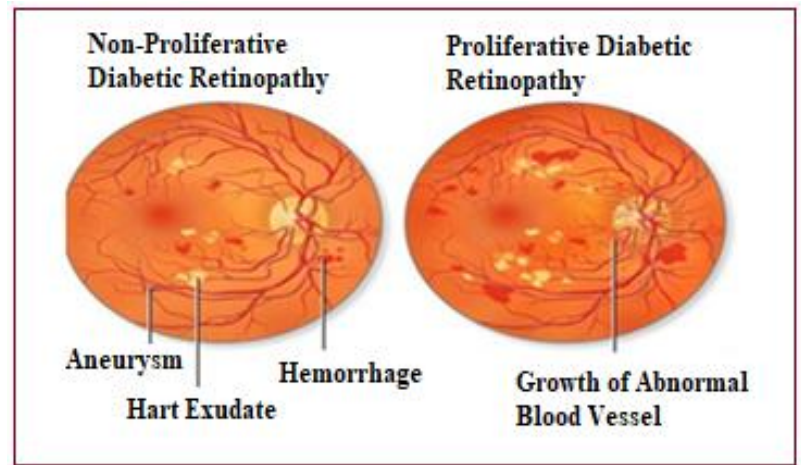

Figure 2: Non-Proliferative (NPDR) and Proliferative Diabetic Retinopathy (PDR).

\subsubsection{Diabetic retinopathy symptoms and risk factors}

Diabetic retinopathy symptoms include dots, dark strings floating in vision, and washed out colors. Diabetes can alter vision in an individual's eye even if he or she does not have retinopathy. Changes in blood sugar change the structure of an eye's lens, and the image on the retina will be out of focus. Blood sugar levels, lipid levels, blood pressure, and duration of diabetes influence the severity of diabetic retinopathy.

\subsubsection{Diabetic Retinopathy Treatment}

The treatment for Diabetic retinopathy is to control the blood sugar level as it will reduce the risk of vision loss and may slow the progression of vision loss. Treatments can vary depending on the stage of the disease and the location of the affected area in the eye. The treatment options are laser surgery, medication injections, and vitrectomy surgery. Laser surgery is the process in which a bright light passes through the cornea, lens, and the vitreous without harm, [16]. The laser shrinks new abnormal blood vessels and reduces macular swelling. Medication injections help to slow the growth and progression of new abnormal blood vessels. Last is the vitrectomy surgery, which is the process in which a microscope 
is used to remove blood and scar tissues that support abnormal vessels in the eye. This surgery removes the vitreous hemorrhage allowing light rays to interact with the retina. The three types of treatment are shown in Figure 3.

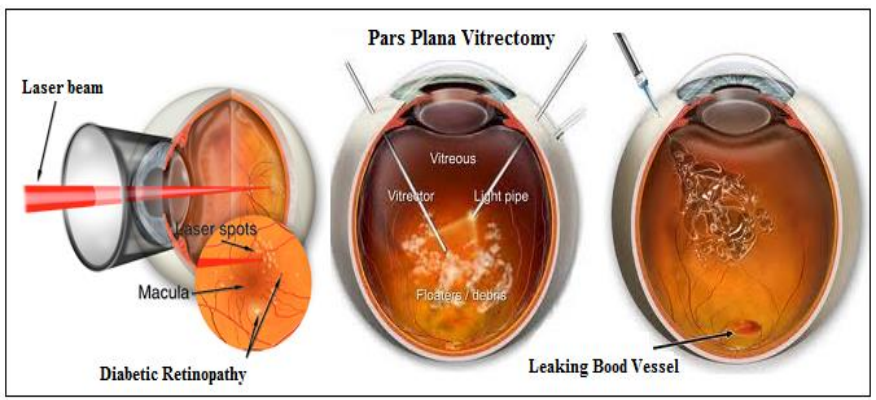

Figure 3: Treatment options: Laser surgery, Vitrectomy surgery and Medication injections.

Vitrectomy surgery often prevents future hemorrhages by removing the vessels that are causing the bleeding. Vitrectomy surgery also removes the damaged tissues, causing the retina to return to its normal position. Medication injections are another way to treat diabetic retinopathy. During this process, medication is injected into the center of the eye (vitreous) to reduce swelling and leakage in the retina, and this method of treatment may improve vision [16 -17].

\subsection{Cataracts}

Cataracts are the process in which the lens of the eye fogs up from time to time. The lens is the bright part of an eye that focuses on images for the retina. In a healthy eye, the transparent lens receives an image that is sent to the retina, which in turn will send signals through the optic nerve to the brain, where the image is interpreted. The lens must be clear, to begin with; if the image is foggy, then the image will have a blurry effect to it. Cataracts can occur in either eye and do not transfer from one eye to the other.

The leading cause of cataracts is age, and their different types are (1) secondary cataracts, which can form post-surgery due to eye problems such as glaucoma; (2) traumatic cataracts, which can be formed after an eye injury; (3) congenital cataracts, which are developed in infants through birth or during childhood; and, finally, (4) radiation cataracts, which can be developed after being exposed to radiation [1820]. The eye with cataract is shown in Figure 4. The primary objective of the lens located behind the pupil is to absorb light onto the retina where the image is created. This lens is contained water and proteins precisely arranged for light to pass through.

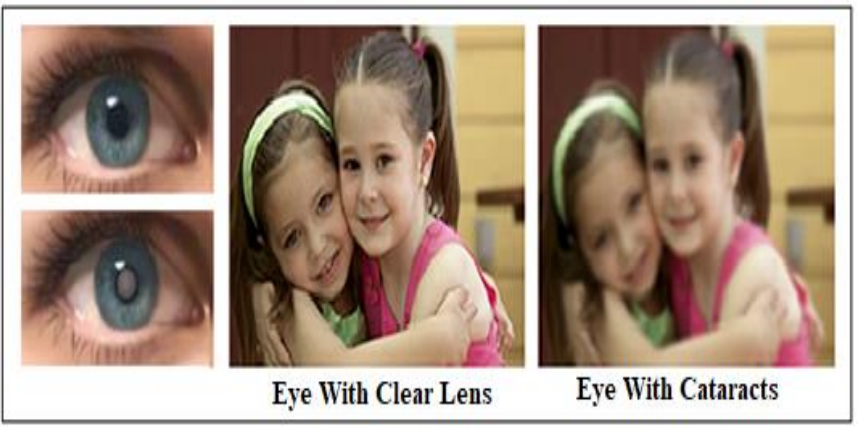

Figure 4: Eye with clear lens and eye has cataracts.

\subsubsection{Cataracts Causes and Risk Factors}

As we age, these proteins may tend to come together to cause fogging in our vision. Risks of cataracts include certain diseases such as diabetes, as well as personal behavior such as alcohol use and smoking.

\subsubsection{Cataracts Symptoms}

The most common symptoms of cataracts are a blurry vision and washed out colors. As individuals progress through the stages of cataracts, less and less light reaches the retina, causing difficulty in vision. Cataracts also affect color vision: colors may begin to seem washed out, and it may become difficult to distinguish between specific colors. Being a victim of cataracts alters sensitivity to light. Bright colors may become too bright and dark colors may become too dark. Diplopia and double vision are symptoms of cataracts when using one eye as a source of vision [20].

\subsubsection{Cataracts Treatments}

The symptoms of cataracts can be improved with the following treatments: eyeglasses, anti-glare sunglasses, and magnifying lenses. If those treatments do not help improve vision, then surgery may be needed. Surgery for cataracts is done to each eye separately with a waiting time of four to eight weeks in between them. The most common surgery for cataracts is known as phacoemulsification. During this type of procedure, the surgeon makes incisions in the eye and breaks up the lens using ultrasound waves. The lens is then taken out of its position and replaced with an intraocular lens (IOL). Another type of cataracts surgery is known as other capsular cataracts surgery. This form of the procedure involves a large incision to remove the foggy lens in one piece rather than breaking it up. These surgeries often eliminate the need for eyewear or contact lenses [19].

\subsection{Macular Degeneration}

Macular Degeneration causes of vision loss among people aged 50 and older. This disease causes damage to the macula, which is a small area near the center of the retina. The retina's central portion is responsible for central vision within the eye. It controls our ability to read a book, recognize faces and colors, and see objects in exquisite detail. The macula is the sensitive part of the retina, which is located at the back of the eye and provides sharp vision. Its primary function is to turn light into signals sent through the optic nerve to the brain where they turned into images. When the macula is damaged, the field of vision may appear blurry, distorted, or dark [21-22]. Macular degeneration is shown below in Figure 5.

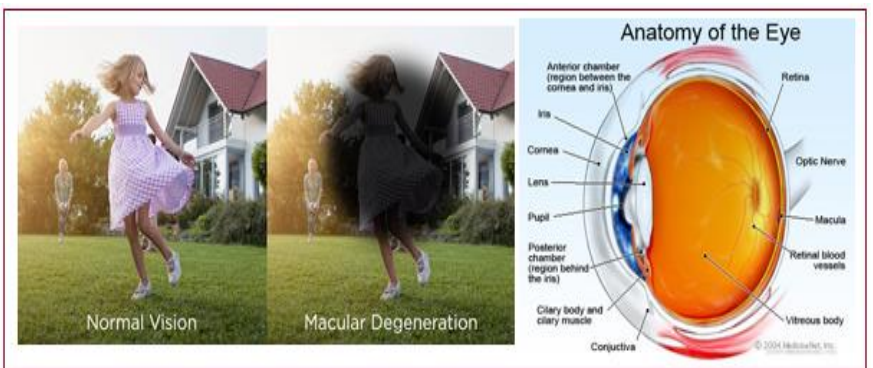

Figure 5: Normal macular degeneration and anatomy of normal eye and loss of central vision. 


\subsubsection{Types of Macular degeneration or (AMD)}

The main types of macular degeneration are dry and wet. Dry degeneration, the most common type, is the process in which cells within the macula gradually become thin or tend to break down, which is known as atrophy. Dry macular degeneration slowly reduces central vision and affects color perception. The condition can go unnoticed if it only occurs in one eye; this is because the healthy eye may compensate for vision loss. Wet macular degeneration, the less common form, can cause more damage to central vision compared to the dry type of AMD, making it more severe. This type of AMD occurs when fluids leak from blood vessels under the macula, blurring central vision and causing rapid vision loss. Symptoms for this form of AMD progress rapidly. The two types of macular degeneration are shown in Figure 6.

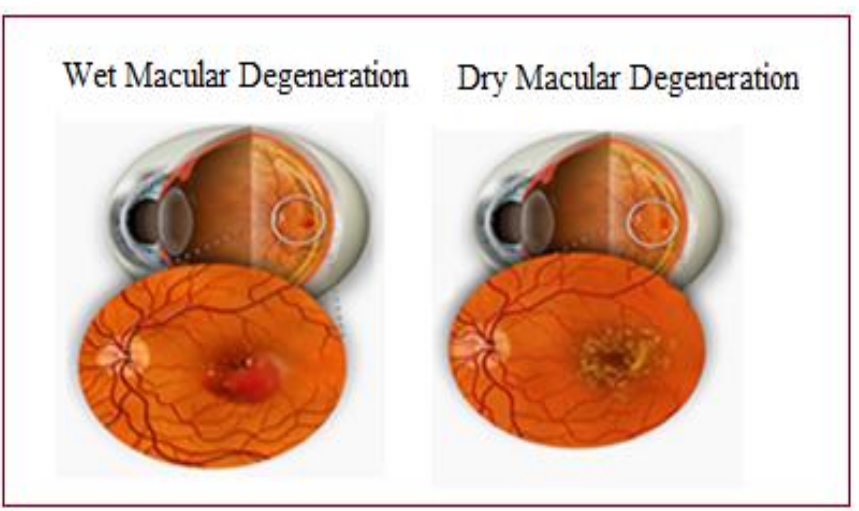

Figure 6: The two types: wet and dry of macular degeneration.

\subsubsection{Symptoms and Risk Factors for (AMD)}

As (AMD) progresses, loss of vision occurs in the form of blurred areas or blank spots in vision. However, the two types of AMD don't necessarily share symptoms. Dry AMD symptoms include blurred vision, a need for increased light for close up vision, and a faded and less vivid appearance of colors. Wet AMD symptoms include loss of central vision, dark spots that appear in vision, and the appearance of the same objects as different sizes between the two eyes. Age is the leading factor for AMD and is more likely to occur after the age of 60. Other factors include smoking, high blood pressure, obesity, and high cholesterol [23]. Figure 7 shows the symptoms of macular degeneration.

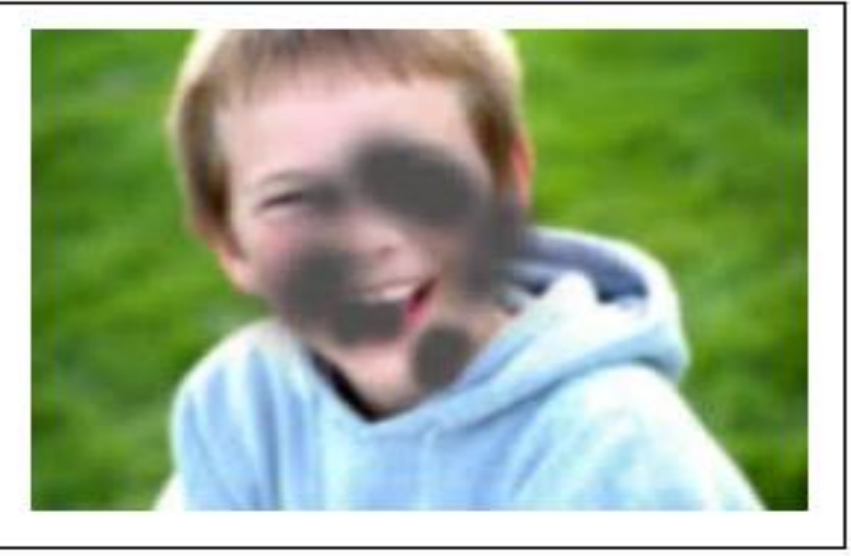

Figure 7: Symptoms of macular degeneration.

\subsubsection{Treatment Options for (AMD)}

When the macula becomes too dry, there is no treatment once it has reached the advanced stage. However, there are methods to possibly delay and prevent intermediate AMD from progressing to the advanced stage in which vision loss occurs. Wet AMD can be treated with the following: antiVEGF medication injections, photodynamic therapy, and laser surgery. None of the treatments listed above will cure the wet AMD, but they do slow further progression of vision loss. AntiVEGF medication injections involve a process in which medications are injected into the eye to block the development and leakage of new abnormal blood vessels. This method of treatment has been proven effective numerous times with patients that have vision loss. Laser surgery involves a highenergy laser light that diminishes the growth of abnormal vessels to prevent problems such as leakage and bleeding. Photodynamic therapy involves a combination of a lightactivated drug called a photosensitizer and a low power cool laser. The photosensitizer is injected into the bloodstream and is absorbed by the abnormal blood vessels, followed by a cold laser that activates the drug causing the abnormal vessels to close off [22-23].

\subsection{Glaucoma}

This kind of disease damages the optic nerve within the eye. The condition becomes worse over time due to pressure build-up in the eye. Glaucoma is a condition that is most likely inherited. In patients suffering from this condition, intraocular pressure damages the optic nerve and causes permanent vision loss. It is a rapidly growing condition if not treated in its early stages and can take effect within a few years, [24]. Figure 8 below shows a healthy eye and an eye with glaucoma.

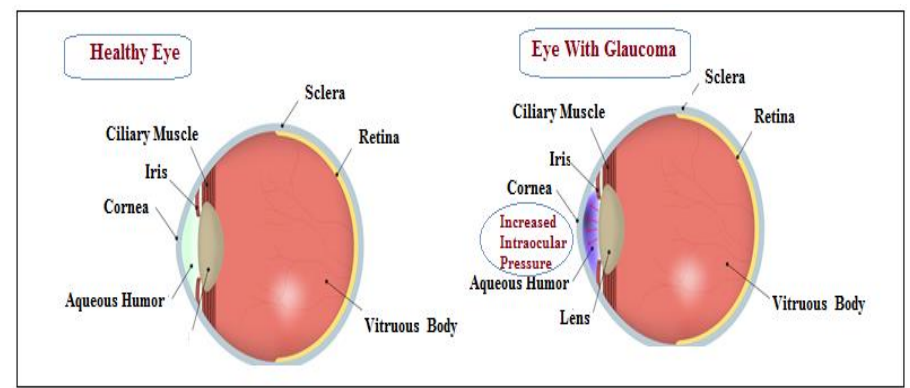

Figure 8: Healthy eye and eye with glaucoma.

\subsubsection{Causes and Symptoms}

Glaucoma occurs in a few different forms, all of which cause vision loss. Primary open angle glaucoma, the most common type, is processed in which fluids freely circulate within the eye and cause pressure build up over time. Another form, known as acute or angle closure glaucoma, develops suddenly and causes pressure to rise rapidly due to the normal flow of fluid being blocked off within the eye. Both forms of glaucoma cause blindness, but each has different symptoms [24-25].

\subsubsection{Open-Angle Glaucoma}

Open-Angle Glaucoma is a form of glaucoma that occurs gradually and painlessly. During this condition, peripheral vision begins to fade away, so that objects are only 
visible through central vision. If no treatment is provided for this condition, central vision begins to fade away as well, leading to complete loss of vision.

\subsubsection{Closed Angle Glaucoma}

Closed-angle glaucoma occurs suddenly. Symptoms of this condition include blurred vision, redness in the eye, severe headache, pain in the eye, extreme weakness, nausea and vomiting, and haziness in the cornea. Open-angle glaucoma and acute glaucoma are shown in Figure 9.

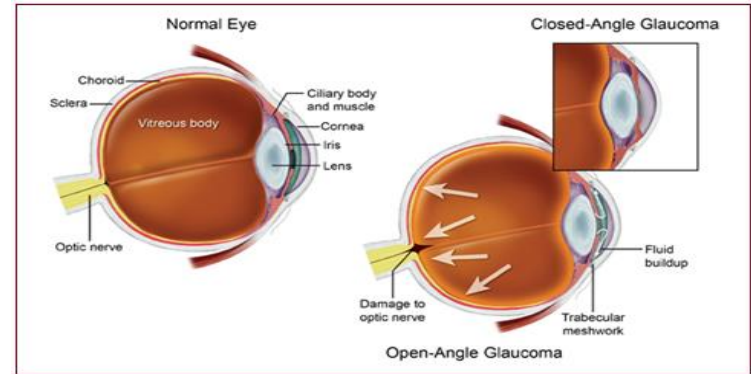

Figure 9: Open-angle glaucoma and acute glaucoma.

\subsubsection{Glaucoma Risk Factors}

Glaucoma is a condition that can affect anyone. However, some people have a higher risk of developing the condition than others: the elderly, people who have had an eye injury, or those with family members afflicted by glaucoma. It is more likely to suffer from glaucoma at a later age, but openangle glaucoma can also be developed suddenly after an eye injury or can be inherited genetically [26].

\subsubsection{Glaucoma Treatment}

There are various methods to slow the progression of the condition, including medication, surgery, and laser treatment. Medicines help to decrease the buildup of pressure within the eye. Surgery may become necessary if medication fails to slow the progression of pressure. Finally, laser treatments help to increase the flow of fluids. There is no exact limit of treatment use as some patients may need lifelong treatments [24]. Figure 10 below shows the procedure of the Glaucoma laser treatment.

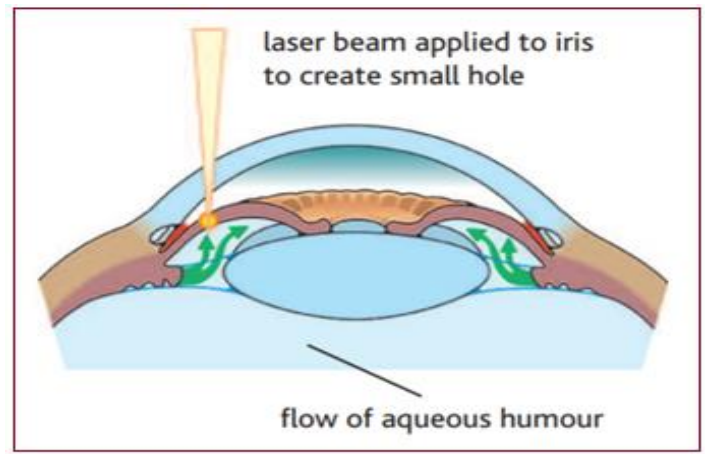

Figure 10: Glaucoma laser treatment.

\section{SMARTPHONE OPHTHALMIC ADAPTERS FOR EYE HEALTH IMAGING DIAGNOSTICS}

The use of smartphones in ophthalmology has dramatically increased; it is now used for a variety of purposes, from evaluating patients and sharing information to registration. New generation smartphones can run multiple advanced applications in medical specialties [27-30]. Most recent smartphones feature the ability to capture high-quality photos through slit lamps, record videos from a microscope, and display images from optical coherence tomography systems and other high-tech devices. Nowadays, ophthalmologists can share images through several communication applications and make diagnostic decisions to help patients with deteriorating vision. Figure 11 shows one use of the smartphone in this field.

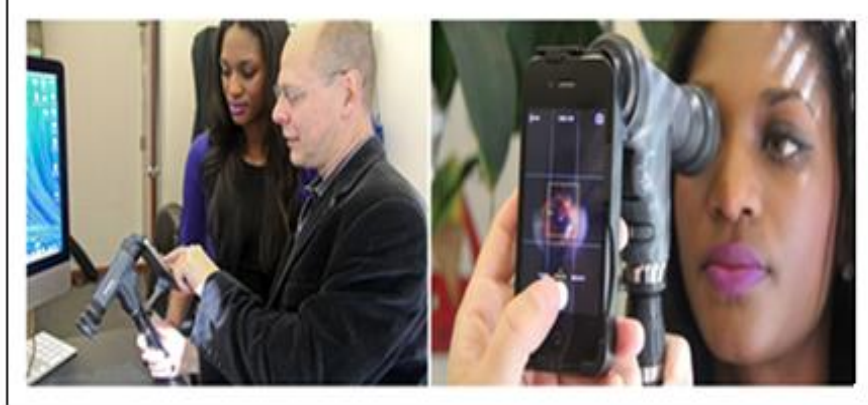

Figure 11: The retina adapter and the examination test.

\subsection{Smartphones Apps in Ophthalmology}

In the field of ophthalmology, smartphone applications can be divided into three groups: those for patients, those for medical or nursing students, and for healthcare professionals. These applications can be used for patient education, assessment, and for physician education and reference. Patient assessment: patient assessment uses several applications, such as near vision cards, color vision plates, penlights, Pupil gauges and rulers, and fluorescein lights to assess visual acuity for patients who cannot read, either because of young age or lack of literacy training. Patient education: Patient education includes visual or verbal materials designed to improve patients' understanding of medical conditions. The most common applications for patient education include Eye handbook and Ikonion.

These applications provide a database of information for common eye conditions, along with clinical features, treatment methods and references to websites for more information on the topic. Physician education: Physician education applications provide ophthalmologists with visual data on changes of the eye throughout different diseases or conditions. These applications also provide questionnaires for common diseases. Physician reference: Smartphone usage is mainly for personal communication, but physicians have been using them as a reference tool. Research shows that $30-40 \%$ of physicians reported using their smartphones for clinical decision support. The use of smartphones during examination allows the physician to share and discuss photos or collect patient data.

\subsection{Evaluation of the Patients' Eyes Using the Smartphone Camera and Smartphone Adapters}

Healthcare professionals are increasingly using smartphone applications. These applications allow patients to monitor their health as well as to connect with healthcare providers. The uses of these devices for ophthalmic photography became ordinary eye care, where there is a growing market of applications and adapters. The new 
generation of smartphones features cameras with a resolution of 5-megapixels and higher, enabling users to capture clear images of the eye. There are several photo adapters available on the market for smartphones to capture both anterior and posterior eye segments. Nowadays, there are a lot of pocketsized adapters such as portable eye examination kit (Peek), DEye, and EyeGo, which improve the lighting and magnification of smartphones, allowing the device to capture even higher quality images of the eye [31-37]. Figure 12 shows eye care professionals examining patients' eyes using different smartphone adapters.

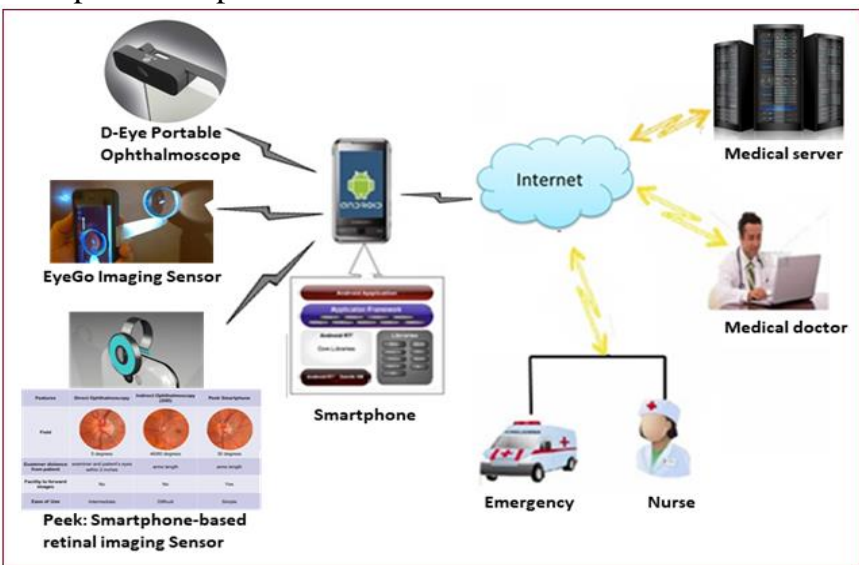

Figure 12: The eye care professionals examine by different smartphone adapters.

\subsubsection{Portable Eye Examination Kit (PEEK)}

The portable Eye Examination Kit (PEEK) consists of smartphone apps and retina adapters that clip onto the device and sync with the PEEK app. This adapter can be used by anyone, anywhere in the world to examine eyes. It is essential as millions of people around the world are not able to get to clinics and hospitals to seek help. These two components allow eye care professionals to examine patients' eyes without ophthalmoscopes, bulky cameras, or other big, expensive equipment. PEEK comes equipped with a clip-on camera adapter that captures high-quality images of the back of the eye and the retina.

It can help healthcare professionals treat people of all ages all around the world who have no access to eye care. PEEK is used for necessary exams, as well as in treating glaucoma, cataracts, diabetic retinopathy, degeneration, nerve disease, macular and other eye conditions. Through the images that healthcare professionals receive from patients, they can determine two common conditions that often cause eye-related problems: diabetes and high blood sugar. Figure 13 shows where the retina adapter is placed onto the device and the examination test.

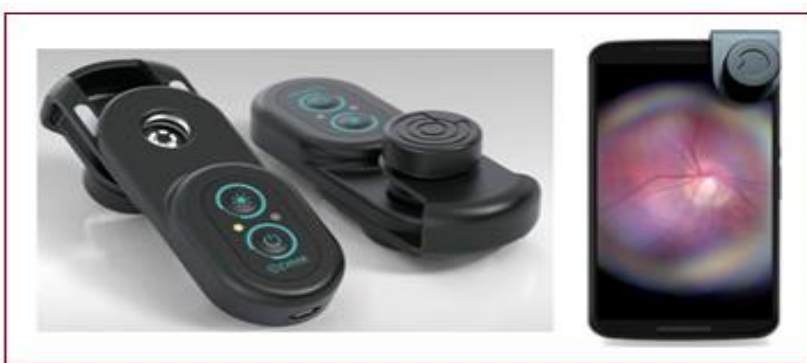

Figure 13: Shows the retina adapter and the examination kit.

\subsubsection{EyeGo Adapter}

EyeGo is an adapter intended to allow ophthalmologists and healthcare specialists to capture high-quality images if the eye using ophthalmic lenses. This adapter consists of two adapters that are added to the smartphone camera and a threedimensional posterior adapter to align the 20 diopter lens with the existing camera on the smartphone. The first adapter captures images of the surface of the eye, while the second adapter focuses light through the pupil on capturing images of the retina. During the process of examination, the right-hand steadies the smartphone and takes an image, and the left hand stabilizes the lens and lifts the upper eyelid. Figure 14 shows the adapter location on the smartphone and the process of the examination.

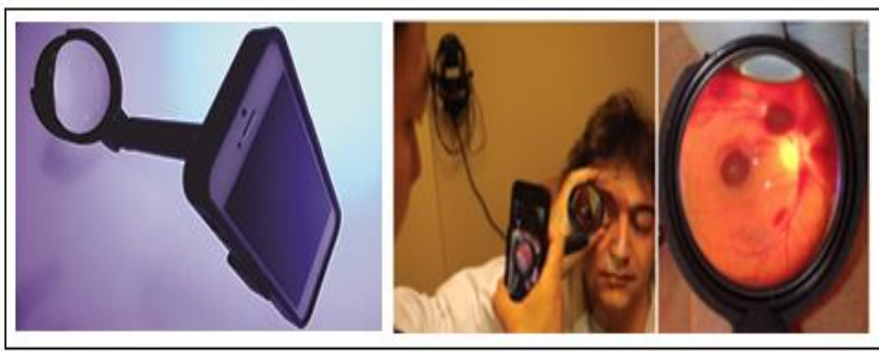

Figure 14: The EyeGo adapter and the image received.

\subsubsection{D-Eye Adapter}

D-Eye Adapter is an adapter consisting of a portable eye and retinal system that fits onto a smartphone creating a retinal camera for evaluation and screening of the eye. It uses LED lights as a light source and requires no extra power, making it an ideal solution for portable diagnostics. Its primary purpose is to examine a patient's retina, optic nerve, or other parts of the eye to determine disorders such as glaucoma, diabetic retinopathy, age-related macular degeneration, hemorrhages, and blood vessel abnormalities.

Patients can capture images of their eye which then can be sent to healthcare professionals for examination. D-Eye adapter has many features and presents excellent advantages compared to EyeGo and Portable Eye Examination Kit (PEEK). D-Eye can record multiple images or videos in a field of view of up to 20 degrees without the use of an external power source, it prescreens anywhere and shares the collected data with other specialists immediately via the web; optional private and secure cloud-based storage system and iOS based applications are also available. Figure 15 shows how eye care professionals examine a patient's eyes by using these smartphone adapters.

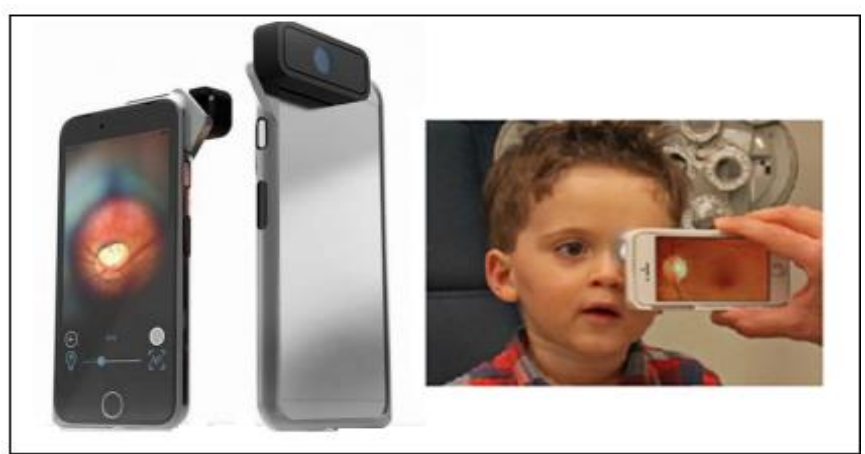

Figure 15: The D-Eye adapter test. 


\section{PERFORMANCE COMPARISON BETWEEN THE THREE ADAPTERS, EYEGO, PEEK, AND D-EYE}

One way to analyze the performance of the smartphone adapters is to compare the three adapter types and their characteristics. This comparison is given in Table 1.

Table 1: Comparison between the three types of Smartphone.

\begin{tabular}{|c|c|c|c|}
\hline Attributes & EyeGo & PEEK & D-Eye \\
\hline Components & $\begin{array}{l}\text { Two adapters and a three- } \\
\text { dimensional posterior } \\
\text { adapter to align the } 20 \\
\text { diopter lens with the } \\
\text { existing camera on the } \\
\text { smartphone. }\end{array}$ & $\begin{array}{l}\text { Retina adapters that clip } \\
\text { onto the device and syncs } \\
\text { to the app. }\end{array}$ & $\begin{array}{l}\text { Portable eye and retinal } \\
\text { system along with an } \\
\text { LED light source that } \\
\text { requires no power. }\end{array}$ \\
\hline Portability & Medium & High & High \\
\hline Purpose & $\begin{array}{l}\text { Intended to capture } \\
\text { images of the eye using } \\
\text { ophthalmic lenses. }\end{array}$ & $\begin{array}{l}\text { Captures images of } \\
\text { patients' eyes without } \\
\text { Ophthalmoscopes }\end{array}$ & $\begin{array}{l}\text { Intended to capture } \\
\text { images of the eye using } \\
\text { ophthalmic lenses. }\end{array}$ \\
\hline Compatibility & Smartphones. & Smartphones. & Smartphones. \\
\hline Examines & $\begin{array}{l}\text { Examines disorders } \\
\text { within the eye. }\end{array}$ & $\begin{array}{l}\text { Glaucoma, Cataracts, } \\
\text { Diabetic retinopathy, } \\
\text { Macular degeneration, } \\
\text { and other eye conditions. }\end{array}$ & $\begin{array}{l}\text { Examines retina, Optic } \\
\text { nerve, and other } \\
\text { disorders such as: } \\
\text { Glaucoma, Cataracts, } \\
\text { Diabetic retinopathy, } \\
\text { Macular degeneration, } \\
\text { and other eye conditions. }\end{array}$ \\
\hline User Friendliness & Medium & High & High \\
\hline Quality of Lens & High quality image & High quality image & High Megapixels image \\
\hline
\end{tabular}

\section{CONCLUSIONS}

Eyes are susceptible and prone to infections and diseases if proper care is not taken. Lowering stress, limiting exposure of eyes to direct light, decreasing usage of electronic devices, and nutritious food can all help reduce the pressures we put on our eyes. In this paper, we have discussed different types of eye diseases, their causes, symptoms, effect on vision and diagnosis. The four eye conditions considered here are diabetic retinopathy, cataracts, macular degeneration, and glaucoma. It is essential to treat these diseases at the initial stages, as waiting for the condition to advance might result in permanent damage that cannot be treated. In this current technological era, smartphones with high-quality cameras can be used to diagnose eye diseases. The working principle behind this method is, the camera takes a high-quality magnifying picture of the eye and displays the images from optical coherence tomography systems. This allows the doctors to monitor and analyze the cause of the diseases closely and diagnose it accordingly based on the available data. It is essential for the smartphone to capture a high-quality magnifying image for accurate analysis of the diseases. Hence, pocket-sized adapters that improve the magnification and lighting are used to take a clear and sharper image of the eye. At present, there are three types of adaptors available: the Portable Eye Examination Kit (PEEK), EyeGo, and D-Eye adapter.
The functioning and applications of these adapters are discussed in this paper. We have argued that the D-eye adapter shows the best performance when compared to D-Eye and PEEK. Currently, advanced research is being undertaken to develop smartphone apps and adapters that be more effective and extensively used in the medical field.

\section{REFERENCES}

[1] Majumder, Sumit, and M. Jamal Deen,"Smartphone sensors for health monitoring and diagnosis." Sensors, 19.9,2164, (2019).

[2] Moradian, Siamak, and Sare Safi, "Application of mobile phones in ophthalmology" Journal of ophthalmic \& vision research 10, no. 2 (2015).

[3] Chhablani, Jay, Simon Kaja, and Vinay A. Shah. "Smartphones in ophthalmology", Indian journal of ophthalmology 60, no. 2, (2012).

[4] Lord, Ron K., Vinay A. Shah, Ashley N. San Filippo, and Rohit Krishna, "Novel uses of smartphones in ophthalmology" Ophthalmology no. 6 (2010): 1274-1274.

[5] Brucker, Julie, et al., "Odysight: A Mobile Medical Application Designed for Remote Monitoring A Prospective Study Comparison with Standard Clinical Eye Tests", Ophthalmology and therapy 8.3 (2019): 461-476.

[6] Zvornicanin, Edita, Jasmin Zvornicanin, and Bahrudin Hadziefendic. "The use of smart phones in ophthalmology", Act Informatics Medical 22, no. 3 (2014).

[7] Bastawrous, A., R. C. Cheeseman, and A. Kumar. "IPhones for eye surgeons", (2012): 343.

[8] Silva, Paolo S., Jerry D. Cavallerano, Lloyd M. Aiello, and Lloyd Paul Aiello. "Telemedicine and diabetic retinopathy: moving beyond retinal screening", Archives of ophthalmology129, no. 2 (2011): 236-242. 
[9] Russo, Andrea, Francesco Morescalchi, Ciro Costagliola, Luisa Delcassi, and Francesco Semeraro ", A novel device to exploit the smartphone camera for fundus photography." Journal of ophthalmology, (2015).

[10] Ting, Daniel SW, Mei Ling Tay-Kearney, and Yogesan Kanagasingam. "Light and portable novel device for diabetic retinopathy screening", Clinical \& experimental ophthalmology 40 , no. 1, (2012).

[11] Vyas, Hitesh J. "Smartphone Ophthalmic Surgery recording for erecords and education", Journal of Education Technology in Health Sciences 3, no. 1 (2016): 13-15.

[12] Ludwig, Cassie A., Somasheila I. Murthy, Rajeev R. Pappuru, Alexandre Jais, David J. Myung, and Robert T. Chang ",A nove smartphone ophthalmic imaging adapter: user feasibility studies in Hyderabad, India." Indian journal of ophthalmology64, no. 3 (2016): 191.

[13] Kambiz Negahban, "Diabetic \& Hypertensive Retinopathy", 2009.Available:http://www.eastoneye.com/diabetic-hypertensiveretinopathy.aspx.

[14] Jesse Vislisel and Thomas Oetting, "Diabetic retinopathy: from one medical student to another," 2010. Available: http://webeye. ophth.uiowa.edu/eyeforum/tutorials/Diabetic-Retinopathy-MedStudents/index.htm

[15] Madeline R. Vann, MPH, "Understanding Diabetic Retinopathy",2015. https://www.everydayhealth.com/hs/diabeteseye-health/ understanding-diabetic-retinopathy/.

[16] Kierstan Boyd, "What Is Diabetic Retinopathy?" 2017. Available: https://www.aao.org/eye-health/diseases/what-is-diabeticretinopathy.

[17] Marilyn Haddrill, "Treatment of Diabetic Retinopathy, and Macular Edema", 2017.

[18] National Eye Institute (NEI), "Facts about Cataract", 2009.Available:https://nei.nih.gov/health/cataract/cataract_fact.

[19] Gretchyn Bailey, Vance Thompson, "Cataracts: Common Types, Causes, and Symptoms", 2016. http:// www.ncascade /com/ services /cataract-surgery.

[20] Joseph Bennington-Castro, Robert Jasmer, "What is a cataract", 2016. http://www.specstore.org/eye-health/cataracts?Next =\&id 3.

[21] Burlina, P., et al. "Unsupervised Deep Novelty Detection: Application to Muscle Ultrasound and Myositis Screening", 2019 IEEE 16th International Symposium on Biomedical Imaging. IEEE, 2019

[22] American Macular Degeneration Foundation, "Macular Degeneration Treatments" 2017. https://www.macular.org/ treatments.
[23] Marilyn Haddrill, "What Is Age-Related Macular Degeneration?”, 2018 https://www.allaboutvision.com/ conditions/amd.htm.

[24] Andrew Dahl, "What are glaucoma symptoms and signs", 2018 https://www.medicinenet.com/glaucoma/article.htm.

[25] Yang, Diya, and Robert N. Weinreb. "Time to Eliminate "Normal Tension", in Primary Open-Angle Glaucoma." Intraocular and Intracranial Pressure Gradient in Glaucoma. Springer, Singapore, 2019.

[26] Ramulu, Pradeep Y., et al. "What is a falls risk factor? Factors associated with falls per time or per step in individuals with glaucoma", Journal of the American Geriatrics Society 67.1 (2019): 87-92

[27] Chhablani, Jay, Simon Kaja, and Vinay A. Shah. "Smartphones in ophthalmology", Indian journal of ophthalmology 60, no. 2 (2012): 127.

[28] Jill Goetz, "UA Engineering Vision Researcher Turns Smartphones into Eye-Screening Tools", 2014. https://uanews.arizona.edu/ story / uaengineering-turns-smartphones-into-eye-screening-tools.

[29] Moradian, Siamak, and Sare Safi, "Application of mobile phones in ophthalmology", Journal of ophthalmic \& vision research 10, no. 2 (2015).

[30] Lakshminarayanan, Vasudevan, John Zelek, and Annette McBride, "Smartphone Science in Eye Care and Medicine", Optics and Photonics News 26, no. 1 (2015): 44-51.

[31] Roy, Somak, et al. "Smartphone adapters for digital photomicrography", Journal of pathology informatics 5 (2014).

[32] Giardini, Mario E., et al. "A smartphone based ophthalmoscope", In Engineering in Medicine and Biology Society (EMBC), 2014 36th Annual International Conference of the IEEE, pp. 2177-2180. IEEE 2014

[33] Maryna Koberidze, "Eye Exams On-the-Go with PEEK", Maryna Koberidze, "Eye Exams On-the-Go with PEEK", 2017. https://medium.com /innovate4health/eye-exams-on-the-go-with-peek$7354 \mathrm{dbc7a6a}$.

[34] Giardini, Mario Ettore. "The Portable Eye Examination Kit: Mobile phones can screen for eye disease in low-resource settings", IEEE pulse 6 , no. 6 (2015): $15-17$

[35] Robert Chang and David Myung, "Advances in Smartphone Photography of the Eye", cataract \& refractive surgery today Europe, September 2014

[36] Ben Coxworth, "EyeGo adapters let you perform eye exams with a smartphone", 2014.

[37] David Nield, "A new smartphone attachment can perform eye exams from anywhere in the world", 2015. Available: https://www. sciencealert.com/a-new-smartphone-attachment-that-could-save-you-atrip-to-the-eye-doctor. 